\title{
Near-Capacity Code Design for Entanglement-Assisted Classical Communication over Quantum Depolarizing Channels
}

\author{
Zunaira Babar, Soon Xin Ng, and Lajos Hanzo
}

\begin{abstract}
We have conceived a near-capacity code design for entanglement-assisted classical communication over the quantum depolarizing channel. The proposed system relies on efficient near-capacity classical code designs for approaching the entanglement-assisted classical capacity of a quantum depolarizing channel. It incorporates an Irregular Convolutional Code (IRCC), a Unity Rate Code (URC) and a soft-decision aided Superdense Code (SD), which is hence referred to as an IRCCURC-SD arrangement. Furthermore, the entanglement-assisted classical capacity of an $N$-qubit superdense code transmitted over a depolarizing channel is invoked for benchmarking. It is demonstrated that the proposed system operates within $0.4 \mathrm{~dB}$ of the achievable noise limit for both 2-qubit as well as 3-qubit SD schemes. More specifically, our design exhibits a deviation of only 0.062 and 0.031 classical bits per channel use from the corresponding 2-qubit and 3-qubit capacity limits, respectively. The proposed system is also benchmarked against the classical convolutional and turbo codes.
\end{abstract}

Index Terms-Entanglement-assisted classical communication, superdense coding, irregular convolutional codes, EXIT charts, near-capacity design.

\section{INTRODUCTION}

$\mathbf{E}$ NTANGLEMENT-ASSISTED classical capacity [1] sets the ultimate capacity limit on the reliable transmission of classical information over a noisy quantum channel, when an unlimited amount of noiseless entanglement is shared between the sender and the receiver. The corresponding classicalquantum-classical transmission model, whereby classical information is transmitted over a quantum channel with the aid of the Superdense (SD) coding protocol, is depicted in Fig. 1. Here, Alice intends to transmit her 2-bit classical message $x$ to Bob using a 2-qubit maximally entangled state $\left|\psi_{x}\right\rangle^{A B}$, where $A$ denotes the information qubit, while $B$ is a preshared entangled qubit transmitted over a noiseless channel. The classical message $x$ is encoded (block $\mathcal{E}$ of Fig. 1) into the corresponding quantum state using the 2-qubit Superdense (2SD) coding protocol of [2]. The processed qubit $A^{\prime}$ is passed through a quantum depolarizing channel, which is denoted

Manuscript received January 13, 2013; revised July 11, October 23, and October 27, 2013. The editor coordinating the review of this paper and approving it for publication was A. Thangaraj.

The authors are with the School of Electronics and Computer Science, University of Southampton, SO17 1BJ, United Kingdom (e-mail: \{zb2g10, sxn, lh\}@ecs.soton.ac.uk).

The financial support of the European Union under the auspices of the CONCERTO project, as well as that of the European Research Council under its Advanced Fellow grant, is gratefully acknowledged.

Digital Object Identifier 10.1109/TCOMM.2013.111013.130035

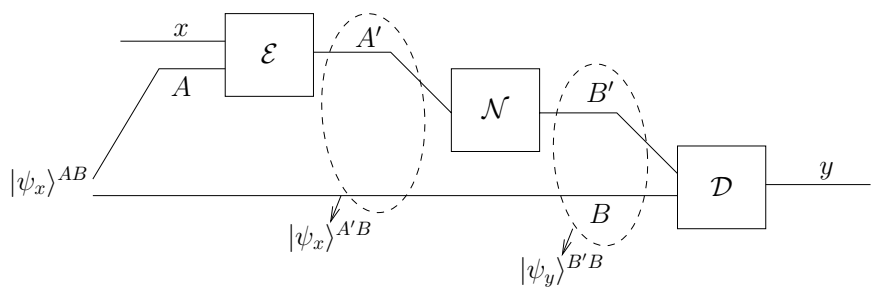

Fig. 1. Classical-quantum-classical transmission model employing 2-qubit SD.

as $\mathcal{N}^{A^{\prime} \rightarrow B^{\prime}}$. Here, $\mathcal{N}^{A^{\prime} \rightarrow B^{\prime}}$ can be viewed as a Completely Positive Trace-Preserving (CPTP) mapping, which maps a state $\rho$ onto a linear combination of itself and the maximally entangled state. More explicitly, for a depolarizing probability $p$, this mapping is given by [3]:

$$
\mathcal{N}_{p}(\rho)=(1-p) \rho+\frac{p}{3} \mathbf{X} \rho \mathbf{X}+\frac{p}{3} \mathbf{Y} \rho \mathbf{Y}+\frac{p}{3} \mathbf{Z} \rho \mathbf{Z},
$$

where $\mathbf{X}, \mathbf{Y}$ and $\mathbf{Z}$ are Pauli matrices. The receiver Bob performs symbol-by-symbol Bell-basis measurement [3], [4] (block $\mathcal{D}$ of Fig. 1) on the received state $\left|\psi_{y}\right\rangle^{B^{\prime} B}$, yielding the 2-bit classical message $y$. Thus, the overall transmission model reduces to a classical discrete-memoryless channel for which the resultant entanglement-assisted classical capacity is quantified as follows [1], [5]:

$$
C_{2 s d}=2+(1-p) \log _{2}(1-p)+p \log _{2}(p / 3) \text { cbits/use }{ }^{1}
$$

This transmission model was extended to a distributed network in [6], whereby the 2SD scheme of [2] was generalized to an $N$-particle system with the aid of an $N$-qubit maximally entangled state. The resultant protocol facilitates the receiver to detect messages from $(N-1)$ users with the aid of a single maximally entangled quantum state as well as a single quantum measurement at the cost of a reduced entanglementassisted classical capacity.

In this treatise, we exploit the efficient near-capacity classical code designs of [7], [8] for the sake of approaching the aforementioned entanglement-assisted classical capacity of the depolarizing channel. More explicitly, we have proposed a superdense coding based near-capacity design for entanglement-assisted classical communication over a depolarizing channel, which incorporates a classical Irregular Convolutional Code (IRCC) and a Unity Rate Code (URC). We have also introduced a soft-decision aided SD decoder

\footnotetext{
${ }^{1}$ Classical bits per channel use.
} 
for facilitating iterative decoding. The proposed scheme is intrinsically amalgamated both with 2-qubit as well as 3qubit superdense codes and it is benchmarked against the corresponding entanglement-assisted classical capacity. Based on our simulation results, the former exhibits only a slight deviation of 0.062 cbits/use with respect to the entanglementassisted capacity, while the latter operates at 0.031 cbits/use from the attainable capacity. In terms of convergence threshold, both schemes operate within $0.4 d B$ of the maximum noise limit.

The rest of the paper is organized as follows. We commence by reviewing the entanglement-assisted classical capacity of $N$-qubit SD in Section II. We then introduce our system model in Section III. This is followed by our near-capacity design, which is detailed in Section IV. Finally, our simulation results are discussed in Section V, while our conclusions are offered in Section VI.

\section{Entanglement-Assisted Classical Capacity of $N$-Qubit SuPERDENSE CODE}

The entanglement-assisted classical capacity of 2SD over a depolarizing channel given by Eq. (2) has been derived in [1], [5] based on its equivalence to a 4-ary discrete classical channel. In this section, we will generalize it to $N$-qubit SD by exploiting the well-known equivalent $M$-ary classical channel model $\left(M=2^{N}\right)$.

Let us recall that the capacity $C$ of a classical channel is equivalent to the maximum value of the conveyed information $I(x, y)$ [9], i.e. we have:

$$
C=\max _{P(x)} I(x, y)=\max _{P(x)}[H(y)-H(y \mid x)],
$$

where $H$ is the classical entropy function. Since $C$ is maximized for equiprobable source symbols, the capacity of an $M$-ary classical channel is given by:

$$
C=\log _{2} M-H(y \mid x),
$$

which is further defined as follows [10], [11]:

$C=\log _{2} M+\mathrm{E}\left[\sum_{m=0}^{M-1} P\left(y \mid x=x^{(m)}\right) \log _{2} P\left(y \mid x=x^{(m)}\right)\right]$,

using Eq. (10) and (11) of [10]. Here E[.] is the expectation (or time average) of $y$ and $x^{(m)}$ is the hypothetically transmitted classical message for $m \in\{0,1, \ldots, M-1\}$.

Based on Eq. (5), the capacity of $N$-qubit SD coding relying on a single noiseless pre-shared entangled qubit may be readily expressed as:

$$
C_{N s d}=\frac{N+\sum_{m=0}^{M-1} P\left(y \mid x=x^{(m)}\right) \log _{2} P\left(y \mid x=x^{(m)}\right)}{N-1},
$$

where $P(y \mid x)$ denotes the transition probabilities of the induced classical channel ${ }^{2}$.

Since symbol-by-symbol measurements performed at the 2-qubit superdense decoder reduces the transmission model

\footnotetext{
${ }^{2}$ Due to the time-invariant nature of $P(y \mid x)$, the average information is the same as the instantaneous value. The expectation operation of Eq. (5) can therefore be ignored.
}

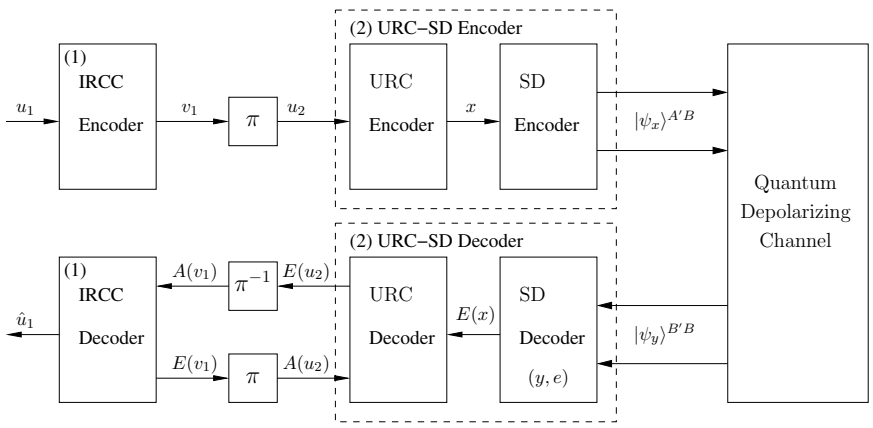

Fig. 2. Schematic of the proposed IRCC-URC-SD classical-quantum communication system.

of Fig. 1 to a 4-ary classical channel, its channel transition probabilities are given by:

$$
P\left(y \mid x=x^{(m)}\right)= \begin{cases}1-p, & \text { if } E=0 \\ p / 3, & \text { if } E \in\{1,2,3\}\end{cases}
$$

where $m \in\{0,1,2,3\}$. Furthermore, $E$ is the decimal equivalent of the $N$-bit classical error $e$, which is induced by the depolarizing channel characterized in Eq. (1). More specifically, the $N$-bit classical error $e=\left[e_{0}, \ldots, e_{i}, \ldots, e_{N-1}\right]$ relates the $i^{\text {th }}$ bit of $x=\left[x_{0}, \ldots, x_{i}, \ldots, x_{N-1}\right]$ to that of $y=\left[y_{0}, \ldots, y_{i}, \ldots, y_{N-1}\right]$ as follows:

$$
y_{i}=x_{i} \oplus e_{i} \text { or } e_{i}=y_{i} \oplus x_{i} .
$$

Similarly, symbol-by-symbol measurements performed at the 3-qubit superdense decoder reduces the overall transmission to an 8-ary classical channel, whose transition probabilities can be readily worked out as follows:

$$
P\left(y \mid x=x^{(m)}\right)= \begin{cases}(1-p)^{2}+p^{2} / 9, & \text { if } E \in\{0\} \\ (1-p)(p / 3)+p^{2} / 9, & \text { if } E \in\{2,3,6,7\} \\ 2(1-p)(p / 3), & \text { if } E \in\{4\} \\ 2 p^{2} / 9, & \text { if } E \in\{1,5\},\end{cases}
$$

where we have $m \in\{0,1, \ldots, 7\}$.

\section{System ModeL}

In this section we will present the architecture of our proposed classical-quantum communication system, which is designed for approaching the entanglement-assisted classical capacity of the $N$-qubit superdense code with the aid of EXtrinsic Information Transfer (EXIT) charts [7], [12], [13]. Fig. 2 shows the general schematic of the proposed system, which employs an Irregular Convolutional Code (IRCC) [14], [15] for achieving the near-capacity performance. Furthermore, a symbol-based recursive Unity Rate Code (URC) having a generator polynomial of $G(D)=\frac{1}{1+D}$ [7] is used as a precoder for reaching the $(1,1)$ point of perfect decoding convergence in the EXIT chart [16]. Since the resultant system of Fig. 2 has three serially concatenated stages, we would need two 3-dimensional EXIT charts, which is cumbersome to handle. Hence, we amalgamated our Superdense code (SD) with the symbol-based URC, which hence constitutes an amalgamated inner component, while the bit-based IRCC is our outer component. 
At the transmitter, the system is fed with classical bits $\left\{u_{1}\right\}$, which are encoded by an IRCC encoder. The IRCCencoded bits $\left\{v_{1}\right\}$ of Fig. 2 are then interleaved $(\pi)$, yielding the permuted bit stream $\left\{u_{2}\right\}$, which is converted to symbols ${ }^{3}$ and fed to the URC encoder of Fig. 2. Classical to quantum domain conversion then takes place at the SD encoder, which maps the classical symbols $x$ onto the orthogonal quantum states $\left|\psi_{x}\right\rangle^{A^{\prime} B}$ using the maximally entangled state $\left|\psi_{x}\right\rangle^{A B}$, as discussed in Section I. Hence, the SD encoder has a function similar to that of the classical PSK/QAM bit-to-symbol mapper, which maps several classical bits onto a complex-valued phasor for communication using the classical electromagnetic waves. The qubits of the resultant quantum state are then serially transmitted over the quantum depolarizing channel ${ }^{4}$.

At the receiver, iterative decoding is invoked for exchanging extrinsic information between the inner (URC-SD) and outer (IRCC) decoders. Here the notations $A(b)$ and $E(b)$ refer to the a priori and extrinsic probabilities of $b$, where we have $b \in\left\{v_{1}, u_{2}, x\right\}$, which are exploited for achieving decoding convergence to a vanishingly low BER. The SD decoder converts the received orthogonal states $\left|\psi_{y}\right\rangle^{B^{\prime} B}$ to classical symbols $y$ by performing a joint measurement in the orthonormal basis. It must be highlighted here that a conventional SD decoder yields the hard-decision outputs. Instead, here we conceive a soft-decision SD decoder, which computes the corresponding extrinsic probability $E(x)$ for the transmitted classical symbol $x$, as follows:

$$
E(x) \approx P(y \mid x),
$$

where $P(y \mid x)$ is given by Eq. (7) and (9) for the 2-qubit and 3 -qubit schemes, respectively.The soft output $E(x)$ is then fed into the URC MAP decoder, which engages in iterative decoding with the IRCC decoder.

\section{NEAR-CAPACITY DESIGN}

\section{A. EXIT Charts}

EXIT charts [7], [12], [13] are capable of visualizing the convergence behaviour of iterative decoding schemes by exploiting the input/output relations of the constituent decoders in terms of their average Mutual Information (MI) transfer characteristics. In the context of our proposed model of Fig. 2, the EXIT chart visualizes the exchange of the following four MI terms:

1) average a priori MI between $u_{2}$ and $A\left(u_{2}\right): I_{A\left(u_{2}\right)}$,

2) average a priori MI between $v_{1}$ and $A\left(v_{1}\right): I_{A\left(v_{1}\right)}$,

3) average extrinsic MI between $u_{2}$ and $E\left(u_{2}\right): I_{E\left(u_{2}\right)}$, and

4) average extrinsic MI between $v_{1}$ and $E\left(v_{1}\right): I_{E\left(v_{1}\right)}$.

Here, $I_{A\left(u_{2}\right)}$ and $I_{E\left(u_{2}\right)}$ constitute the EXIT curve of the inner decoder, while $I_{A\left(v_{1}\right)}$ and $I_{E\left(v_{1}\right)}$ yield the EXIT curve of the outer decoder. For the sake of constructing the inner and outer EXIT curves, the a priori information, $A\left(u_{2}\right)$ and $A\left(v_{1}\right)$ respectively, is modeled using a Gaussian distribution, having a mean of zero and a variance of $\sigma_{A}^{2}$, for a range of

\footnotetext{
${ }^{3}$ Bit-to-symbol convertor is assumed to be inside the URC Encoder block of Fig. 2.

${ }^{4}$ As illustrated earlier in Fig. 1, the processed qubit(s) $A^{\prime}$ is transmitted over the noisy quantum channel, while $B$ is shared between Alice and Bob over a noiseless channel.
}

$I_{A\left(u_{2}\right)}, I_{A\left(v_{1}\right)} \in[0,1]$. The corresponding average extrinsic MI can be formulated as [10], [11]:

$$
I_{E\left(u_{2}\right)}=\log _{2} M+\mathrm{E}\left[\sum_{m=0}^{M-1} E\left(u_{2}^{(m)}\right) \log _{2}\left(E\left(u_{2}^{(m)}\right)\right)\right] \text {, }
$$

and

$$
I_{E\left(v_{1}\right)}=\log _{2} M+\mathrm{E}\left[\sum_{m=0}^{M-1} E\left(v_{1}^{(m)}\right) \log _{2}\left(E\left(v_{1}^{(m)}\right)\right)\right] .
$$

Furthermore, since we are employing symbol-to-bit conversion at the URC decoder, we incorporate binary EXIT charts in our design. This in turn implies that in Eq. (11) and (12) we have $M=2$ and $m \in\{0,1\}$. The resultant inner EXIT function $T_{u_{2}}$ is given by:

$$
I_{E\left(u_{2}\right)}=T_{u_{2}}\left[I_{A\left(u_{2}\right)}, p\right],
$$

and outer EXIT function $T_{v_{1}}$ is as follows:

$$
I_{E\left(v_{1}\right)}=T_{v_{1}}\left[I_{A\left(v_{1}\right)}\right] .
$$

More explicitly, unlike $T_{v_{1}}, T_{u_{2}}$ is a function of the depolarizing probability $p$, since the inner decoder is fed by the channel. Finally, the MI transfer characteristics of both the decoders encapsulated by Eq. (13) and (14) are plotted in the same graph, with the $x$ and $y$ axes of the outer decoder swapped. The resultant EXIT chart is capable of visualizing the exchange of extrinsic MI as a stair-case-shaped decoding trajectory, as the iterations proceed.

\section{B. Near-Capacity IRCC-URC-SD Design}

We have exploited the area property of EXIT charts [17] for designing a near-capacity classical error correction code for our classical-quantum communication system of Fig. 2. According to this property, the area under the EXIT curve of the inner decoder is approximately equal to the attainable channel capacity [17], provided that the channel's input symbols are equiprobable. Since our system model of Fig. 2 transmits classical information over a quantum depolarizing channel, the attainable channel capacity of the system is the entanglementassisted classical capacity given in Eq. (6). However, as mentioned in Section III, symbol-to-bit conversion takes place at the output of the URC decoder. This incurs an inherent capacity loss, which cannot be recovered by any bit-based error correcting code. The capacity loss for both the 2SD and 3SD schemes is quantified in Fig. 3, which compares their bit-based and symbol-based capacities. Here, the bitbased capacity is computed by marginalizing the symbolbased probabilities $P(y \mid x)$ of Eq. (7) and (9) to bit-based probabilities $P\left(y_{i} \mid x_{i}\right)$ for $i \in\{0,1, \ldots, N-1\}$, assuming that the constituent bits are independent. Nevertheless, it must be pointed out that by virtue of being a unity rate code, the URC does not impose any capacity loss, as verified in Fig. 3. The capacity of our inner decoder (URC-SD) is approximately equal to the attainable bit-based entanglement-assisted classical capacity for both 2-qubit and 3-qubit superdense codes. The URC is only invoked for transforming the horizontal EXIT curve of the SD decoder to a slanted one for the sake of improving the scheme's decoding convergence, as detailed in the next section. 


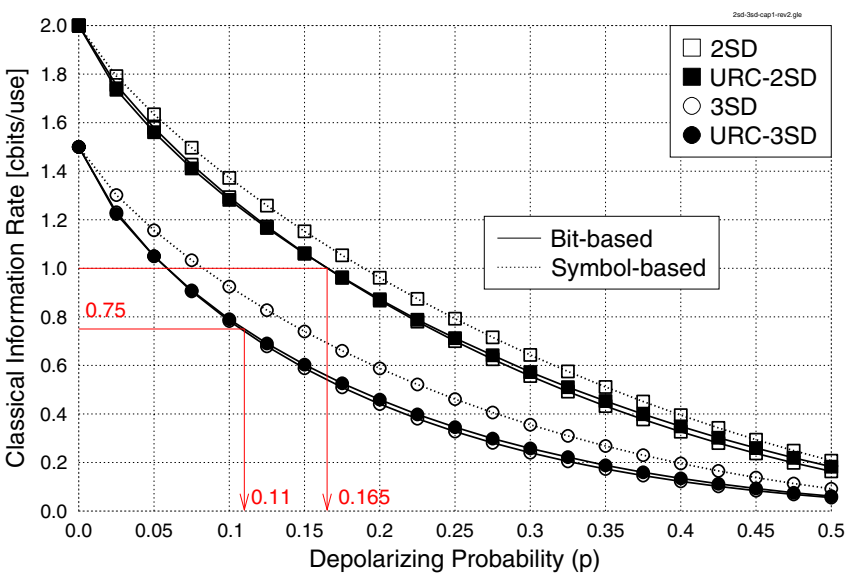

Fig. 3. Classical information rate (cbits/use) versus quantum depolarizing probability for 2-qubit and 3-qubit superdense codes with and without URC.

Furthermore, the area under the EXIT curve of the outer decoder is equivalent to $\left(1-R_{o}\right)$, where $R_{o}$ is its coding rate [17]. Therefore, our near-capacity design aims for creating a narrow, but marginally open tunnel between the EXIT curves of the inner and outer decoders at the highest possible depolarizing probability, which corresponds to the lowest possible SNR for a classical channel. A feasible design option could be to create the EXIT curves of all the possible convolutional codes to find the optimal code $\mathcal{C}$, which gives the best match, i.e. whose EXIT curve yields a marginally open tunnel with the inner decoder's EXIT curve of URC-SD. To circumvent this tedious task, we have invoked the Irregular Convolutional Code (IRCC) of [15], whereby a family of subcodes $\mathcal{C}_{l}$, $l \in\{1,2, \ldots, L\}$, is used for constructing the target code $\mathcal{C}$. Due to its inherent flexibility, the resultant IRCC provides a better match than any single code. Furthermore, for the sake of reducing the encoding and decoding complexity, the family of subcodes $\mathcal{C}_{l}$ is constructed by selecting an $r_{1}$-rate convolutional code $\mathcal{C}_{1}$ as the mother code and obtaining the remaining $(L-1)$ subcodes $\mathcal{C}_{l}$ of rate $r_{l}>r_{1}$ by puncturing the mother code $\mathcal{C}_{l}$. The $l^{\text {th }}$ subcode has a coding rate of $r_{l}$ and it encodes a specifically designed fraction, $\varrho_{l}$, of the original information bits to $\varrho_{l} N_{c}$ encoded bits. Here, $N_{c}$ is the total length of the coded frame. More specifically, for an $L$-subcode IRCC, $\varrho_{l}$ is the $l^{t h}$ IRCC weighting coefficient satisfying the following constraints [14], [15]:

$$
\sum_{l=1}^{L} \varrho_{l}=1, R_{o}=\sum_{l=1}^{L} \varrho_{l} r_{l}, \varrho_{l} \in[0,1], \forall l,
$$

which can be conveniently represented in the following matrix form:

$$
\begin{aligned}
{\left[\begin{array}{cccc}
1 & 1 & \ldots & 1 \\
r_{1} & r_{2} & \ldots & r_{L}
\end{array}\right]\left[\begin{array}{lll}
\varrho_{1} & \varrho_{2} \ldots & \varrho_{L}
\end{array}\right]^{T}=} & {\left[\begin{array}{c}
1 \\
R_{o}
\end{array}\right] } \\
\mathbf{C} \varrho & =\mathbf{d} .
\end{aligned}
$$

In our design, we have employed an IRCC relying on a set of 17 memory-4 convolutional subcodes having 17 different coding rates between 0 and 1 , which was found in [15]. These 17 subcodes are derived such that it covers the complete range of coding rates from 0.1 to 0.9 with a rate-increment of 0.05 , i.e. having rates of $r_{l} \in\{0.1,0.15,0.2, \ldots, 0.85,0.9\}$.

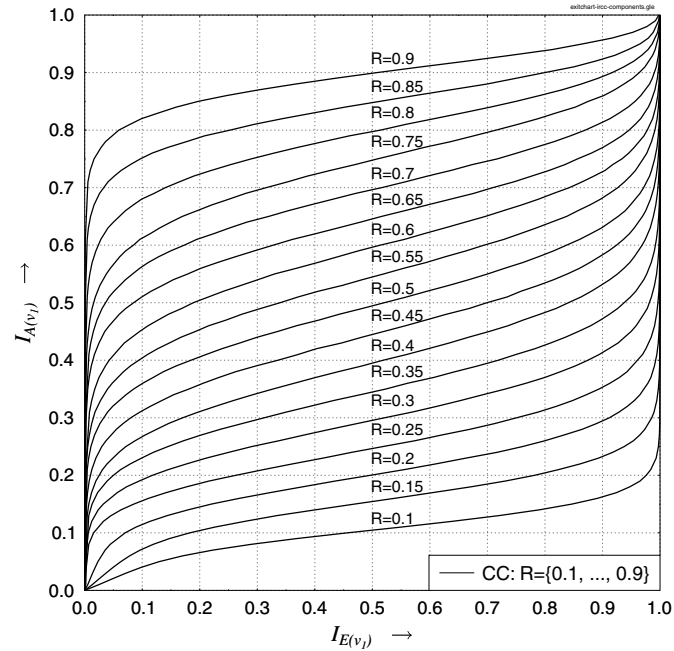

Fig. 4. Normalized outer EXIT curves (inverted) of the 17 IRCC subcodes.

Fig. 4 shows the inverted outer EXIT curves for each of the constituent subcode of the IRCC scheme.

In physically tangible terms, the input bit stream is divided into 17 fractions corresponding to the 17 different-rate subcodes and the specific optimum fractions to be encoded by these codes are found by dynamic programming. More specifically, the EXIT curves of the 17 subcodes, given in Fig. 4, are superimposed onto each other after weighting by the appropriate fraction-based weighting coefficients, which are determined by minimizing the area of the open EXITtunnel. To elaborate a little further, the transfer function of the IRCC is given by:

$$
I_{E\left(v_{1}\right)}=T_{v_{1}}\left[I_{A\left(v_{1}\right)}\right]=\sum_{l=1}^{L} \varrho_{l} T_{v_{1}, l}\left[I_{A\left(v_{1}\right)}\right],
$$

where $T_{v_{1}, l}\left[I_{A\left(v_{1}\right)}\right]=I_{E\left(v_{1}\right), l}$ is the transfer function of the $l^{\text {th }}$ subcode. We employed the curve matching algorithm of [14], [15] for optimizing the weighting coefficients of the IRCC subcodes by ensuring that a narrow, yet open tunnel exists between the EXIT curves of the outer and inner decoder at the highest possible depolarizing probability; thus, guaranteeing that the system has a near-capacity performance.

\section{RESUlts AND Discussions}

Based on the aforementioned approach, we have designed a superdense coding based near-capacity code for entanglementassisted classical communication over the quantum depolarizing channel. Since we intend to design a $1 / 2$-rate system, we have assumed a constant overall coding rate of 0.5 for the IRCC. Fig. 5 shows the normalized EXIT curves for 2SD at a depolarizing probability of 0.15 and using an interleaver length of 30,000 bits. As expected, the EXIT curve of the 2SD decoder is a horizontal straight line. Hence, our URC is used as a precoder, to transform this horizontal EXIT curve into a slanted curve which terminates at the $(1,1)$ point of the EXIT chart; thus, facilitating convergence to an infinitesimally low BER. More specifically, the area under the EXIT curve remains the same, yet reaches the $(1,1)$ point. Furthermore, using the curve matching algorithm of [14], [15], the IRCC 


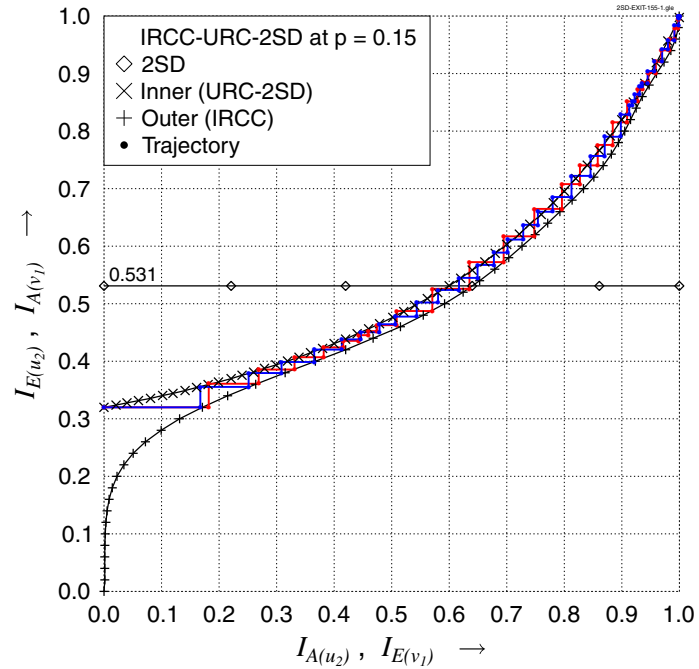

Fig. 5. Normalized EXIT curves of the IRCC-URC-2SD system at a depolarizing probability of 0.15 .

weight vector was optimized to get a narrow open tunnel as evident in Fig. 5. The corresponding weights of the 17 IRCC subcodes are $\varrho^{T}=\left[\begin{array}{lllllll}0 & 0 & 0 & 0.0177 & 0.0145 & 0 & 0.6455\end{array}\right.$ $\left.\begin{array}{llllllllllll}0 & 0 & 0 & 0 & 0.1797 & 0.0580 & 0 & 0 & 0.0105 & 0.0742\end{array}\right]$, where only seven subcodes are activated. The tunnel is narrow, but wide enough for successful convergence, as visualized using the decoding trajectories of Fig. 5. If the depolarizing probability is increased beyond 0.15 , the EXIT curves of the inner and outer decoder crossover, hence closing the tunnel. Thus, the system has a convergence threshold of 0.15 . In other words, it can tolerate depolarizing probabilities upto $p=0.15$, and yet achieve an infinitesimally low BER. However, this would require a high number of iterations between the IRCC and URC-2SD, hence imposing a high complexity.

The coding rate of the designed IRCC-URC-2SD system is $1 \mathrm{cbit} / \mathrm{use}$, since a $1 / 2$-rate IRCC is used. From the bit-based capacity curve of Fig. 3, it can be found that the associated maximum tolerable depolarizing probability is 0.165 . By contrast, the convergence threshold of our system is 0.15 . Thus, it operates only $\left[10 \times \log _{10}\left(\frac{0.165}{0.15}\right)\right]=0.4 \mathrm{~dB}$ within the capacity limit. Alternatively, this discrepancy may also be quantified in terms of the difference in the area under the inner and outer EXIT curves, which corresponds to the normalized capacity loss. The area under the normalized EXIT curve of our URC-2SD scheme is 0.531, whereas that under the IRCC is 0.5 . Thus, the capacity of our IRCC-URC-2SD scheme is only $[0.031 \times 2]=0.062$ cbits/use away from the capacity, when $p=0.15$.

As another example, Fig. 6 shows the EXIT curves for our 3 -qubit SD at a depolarizing probability of 0.1 . The optimized

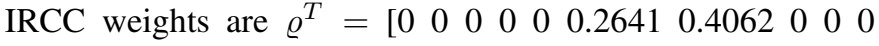
$\left.\begin{array}{lllllllll}0 & 0.1068 & 0.1247 & 0 & 0 & 0 & 0.0982\end{array}\right]$, where only five subcodes are activated. For $p \leq 0.1$, the system successfully converges and the decoding trajectory terminates at the $(1,1)$ point of the EXIT chart. Since our 3SD transmits 1.5 cbits/use and we have used 1/2-rate IRCC, the effective throughput of the designed system is 0.75 cbits/use. The corresponding depolarizing probability according to the bit-based capacity curve of Fig. 3 is 0.11 . Thus, in terms of the depolarizing probability,

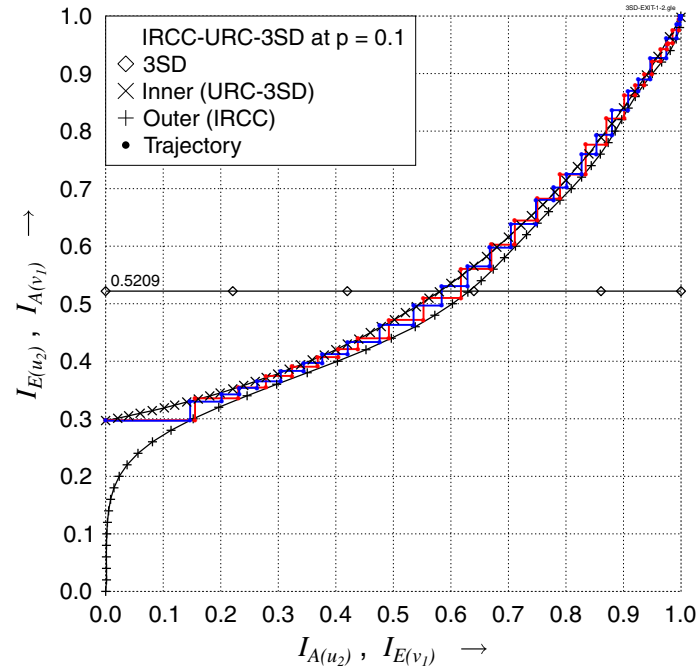

Fig. 6. Normalized EXIT curves of the IRCC-URC-3SD system at a depolarizing probability of 0.1 .

our designed system operates within $\left[10 \times \log _{10}\left(\frac{0.11}{0.10}\right)\right]=0.4$ $\mathrm{dB}$ of the capacity. Furthermore, the area under the normalized EXIT curve of the inner decoder is 0.5209 . The deviation from the capacity curve is therefore $[0.0209 \times 1.5] \approx 0.031$ cbits/use.

We have also evaluated the BER performance curves for both our 2-qubit and 3-qubit based IRCC-URC-SD schemes, which are shown in Fig. 7. As it can be observed, the performance improves upon increasing the number of iterations. More specifically, the 2-qubit system starts to converge to a lower BER, as the number of iterations increases at a depolarizing probability of $p=0.15$. Similarly, the 3-qubit scheme has a threshold of $p=0.1$. Thus, these values match the convergence thresholds predicted using EXIT charts. More explicitly, since the EXIT chart tunnel closes beyond these depolarizing probability thresholds, the system fails to converge, if the depolarizing probability is increased further. Hence, the performance does not improve upon increasing the number of iterations if the depolarizing probability exceeds the threshold. By contrast, when the depolarizing probability is below the threshold, the BER improves at each successive iteration. Here, the trade-off between the complexity imposed and the performance attained comes into play. It should also be noted that the performance improves with diminishing returns at a higher number of iterations. For example, doubling the number of iterations from $I=8$ to $I=16$ for IRCCURC-2SD increases the depolarizing probability by 0.0225 , corresponding to a BER of $10^{-4}$. A further increase to $I=32$ iterations only improves $p$ by around 0.01 at a BER of $10^{-4}$.

To elaborate further on the significance of using an IRCC rather than a conventional 1/2-rate Convolutional Code (CC), we have also conceived a corresponding setup, whereby the IRCC of Fig. 2 is replaced by a memory-4 1/2-rate CC in the proposed IRCC-URC-2SD system. This is synonymous to employing an IRCC with a weighting factor of $\varrho^{T}=\left[\begin{array}{lll}0 & 0 & 0\end{array}\right.$

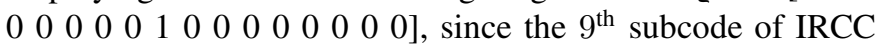
is a $1 / 2$-rate CC. Fig. 8 shows the resultant EXIT curves for $p=0.15$ and $p=0.125$. It can be observed in Fig. 8 that for $p=0.15$, which is the convergence threshold of our IRCC- 


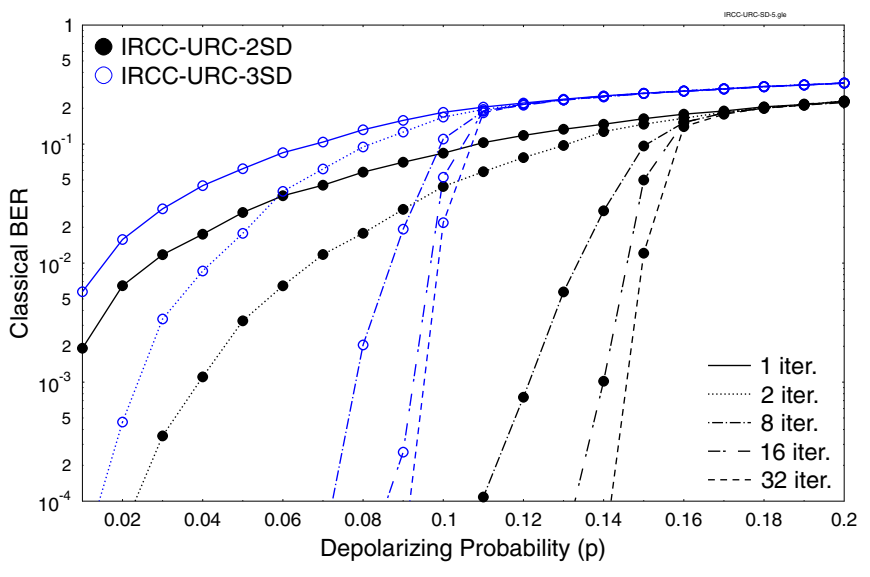

Fig. 7. The BER versus quantum depolarizing probability for IRCC-URC-SD schemes.

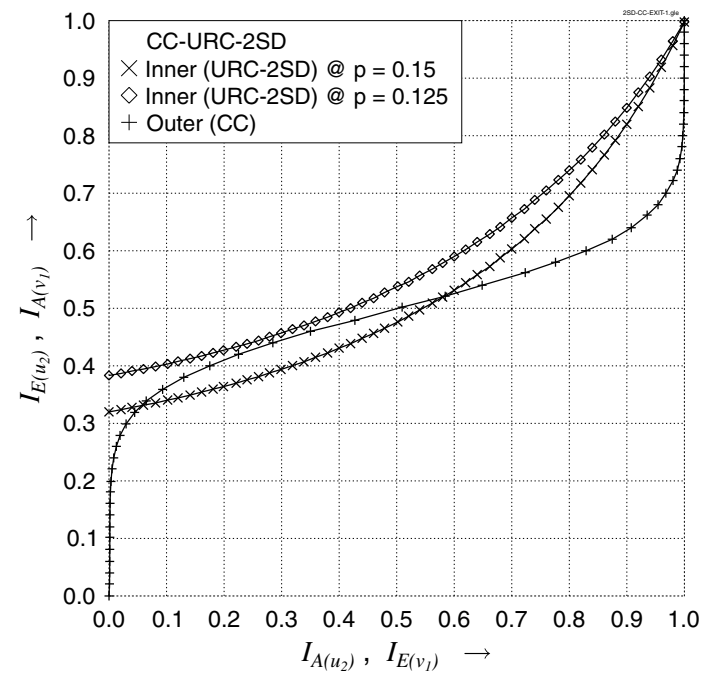

Fig. 8. Normalized EXIT curves of the CC-URC-2SD system .

URC-2SD design, the inner and outer EXIT curves of the CC-URC-2SD scheme exhibit a cross-over. Thus, implying that the CC-URC-2SD configuration fails to converge at $p=$ 0.15 . An open tunnel emerges only when $p$ is decreased to 0.125 . Consequently, the convergence threshold of CC-URC$2 \mathrm{SD}$ is 0.125 , which is lower than that of our near-capacity design of Fig. 5. It must also be pointed out here that the area between the inner and outer EXIT curves at the convergence threshold is wider than Fig. 5. The wider the gap, the higher the capacity loss. Therefore, using a regular CC, rather than an IRCC, yields a poor match between the inner and outer decoders' EXIT curves.

We have further benchmarked the performance of our system against the classical Turbo Code (TC) in Fig. 9. This was achieved by replacing the IRCC-URC unit of Fig. 2 with TC . We have used a memory-3 1/2-rate TC for our comparison, since it invokes 16 states in each iteration, which is the same as the number of states invoked per iteration in our design ${ }^{6}$. The

\footnotetext{
${ }^{5}$ Symbol-to-bit conversion takes place at the output of SD decoder. Consequently, the symbol-based probabilities of Eq. (7) and (9) are converted to bit-based log likelihood ratios (LLRs), assuming that the bits constituting the symbol are independent.

${ }^{6}$ Since a memory- 3 turbo code has two components with $2^{3}$ states, total number of states per iteration are $2 \times 2^{3}=16$. Similarly, a memory-4 IRCC invokes $2^{4}=16$ states per iteration.
}

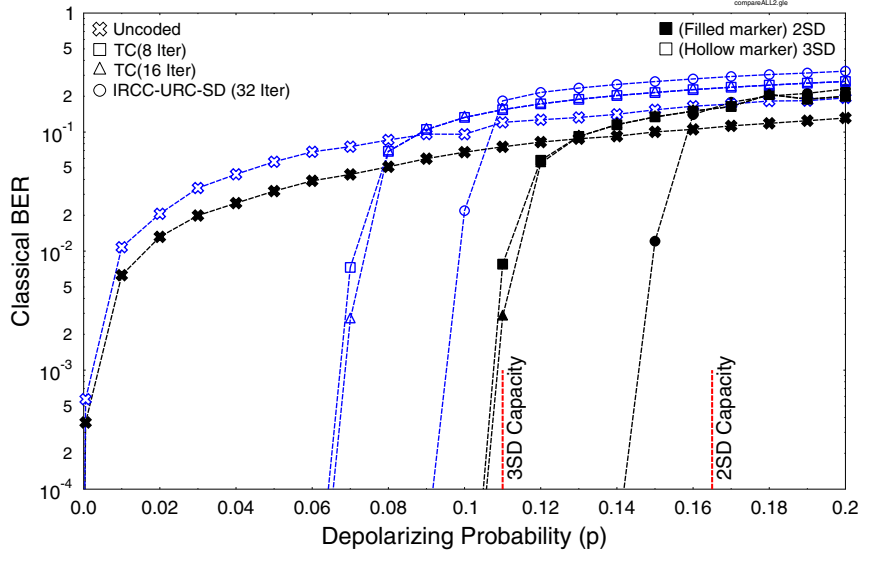

Fig. 9. Comparison of IRCC-URC-SD with TC-SD. 2SD and 3SD are plotted with filled and hollow markers, respectively.

TABLE I

DEVIATION OF TC-SD AND IRCC-URC-SD FROM THE CAPACITY AT A BER OF $10^{-4}$.

\begin{tabular}{|l|c|c|}
\hline & TC-SD & IRCC-URC-SD \\
\hline 2SD & $1.9 \mathrm{~dB}$ & $0.6 \mathrm{~dB}$ \\
3SD & $2.5 \mathrm{~dB}$ & $0.75 \mathrm{~dB}$ \\
\hline
\end{tabular}

uncoded BER curves of our 2SD and 3SD schemes are also plotted in Fig. 9. Furthermore, we have used a sufficiently high number of iterations, i.e. $I=32$, for our designed system to ensure that the system reaches the top right corner of the EXIT chart at a depolarizing probability that is close to the maximum tolerable depolarizing probability. By contrast, $I=16$ iterations were used for TC since it did not yield any appreciable performance improvement, when the number of iterations was increased beyond $I=8$, as evidenced in Fig. 9. Our proposed IRCC-URC-SD system is capable of performing closer to the capacity, hence, outperforming the turbo code for both $2 \mathrm{SD}$ and 3SD. The corresponding deviations from the capacity expressed in terms of $\mathrm{dB}$ at a BER of $10^{-4}$ are tabulated in Table I, where the deviation is the distance from the maximum tolerable depolarizing probability of 0.165 and 0.11 for $2 \mathrm{SD}$ and $3 \mathrm{SD}$, respectively.

\section{CONCLUSIONS}

In this paper, we have proposed an EXIT chart based nearcapacity design for entanglement-assisted classical communication over a quantum depolarizing channel. Our proposed IRCC-URC-SD system of Fig. 2 exploits channel coding operating in the classical domain by serially concatenating an IRCC and a URC with the SD encoder. Furthermore, we have introduced a soft-decision aided superdense decoder facilitating iterative decoding. The EXIT chart of the scheme was used for assisting our design and the achievable information rates were quantified for both 2-qubit and 3-qubit superdense codes. Our BER performance curves confirmed the EXIT chart predictions. Moreover, the designed system outperformed its counterpart relying on the classical convolutional as well as turbo codes. 


\section{REFERENCES}

[1] C. H. Bennett, P. W. Shor, J. A. Smolin, and A. V. Thapliyal, "Entanglement-assisted classical capacity of noisy quantum channels," Phys. Rev. Lett., vol. 83, pp. 3081-3084, Oct 1999.

[2] C. H. Bennett, "Communication via one- and two-particle operators on Einstein-Podolsky-Rosen states," Physical Rev. Lett., vol. 69, no. 20, p. 2881, 1992.

[3] M. A. Nielsen and I. L. Chuang, Quantum Computation and Quantum Information. Cambridge University Press, 2000.

[4] P. Botsinis, S. X. Ng, and L. Hanzo, "Quantum search algorithms, quantum wireless, and a low-complexity maximum likelihood iterative quantum multi-user detector design," IEEE Access, vol. 1, pp. 94122, 2013. Available: http://ieeexplore.ieee.org/xpl/articleDetails.jsp? arnumber $=6515077$.

[5] Z. Shadman, H. Kampermann, C. Macchiavello, and D. Bruß, "Optimal super dense coding over noisy quantum channels," New J. Physics, vol. 12, July 2010.

[6] S. Bose, V. Vedral, and P. L. Knight, "A multiparticle generalization of entanglement swapping," Phys. Rev. A, vol. 57, no. quant-ph/9708004. 2, pp. 822-829, 1998.

[7] L. Hanzo, T. H. Liew, B. L. Yeap, R. Y. S. Tee, and S. X. Ng, Turbo Coding, Turbo Equalisation and Space-Time Coding: EXIT-Chart-Aided Near-Capacity Designs for Wireless Channels, 2nd ed. John Wiley IEEE Press, 2011.

[8] S. X. Ng, J. Wang, and L. Hanzo, "Unveiling near-capacity code design: the realization of Shannon's communication theory for MIMO channels," in Proc. 2008 IEEE International Conference on Communications, pp. $1415-1419$.

[9] C. E. Shannon, "A mathematical theory of communication," The Bell System Technical J., vol. 27, pp. 379-656, July 1948.

[10] J. Kliewer, S. X. Ng, and L. Hanzo, "Efficient computation of EXIT functions for non-binary iterative decoding," IEEE Trans. Commun., vol. 54, pp. 2133-2136, Dec. 2006.

[11] S. X. Ng, O. Alamri, Y. Li, J. Kliewer, and L. Hanzo, "Near-capacity turbo trellis coded modulation design based on EXIT charts and union bounds," IEEE Trans. Commun., vol. 56, pp. 2030-2039, Dec. 2008.

[12] S. ten Brink, "Convergence behaviour of iteratively decoded parallel concatenated codes," IEEE Trans. Commun., vol. 49, pp. 1727-1737, Oct. 2001.

[13] M. El-Hajjar and L. Hanzo, "Exit charts for system design and analysis," IEEE Commun. Surveys \& Tutorials, pp. 1-27, 2013.

[14] M. Tüchler and J. Hagenauer, "EXIT charts of irregular codes," in Proc. 2002 Conference on Information Science and Systems, pp. 465-490.

[15] M. Tüchler, "Design of serially concatenated systems depending on the block length," IEEE Trans. Commun., vol. 52, pp. 209-218, Feb. 2004.

[16] K. R. Narayanan, "Effect of precoding on the convergence of turbo equalization for partial response channels," IEEE J. Sel. Areas Commun. vol. 19, pp. 686-698, Apr. 2001.

[17] A. Ashikhmin, G. Kramer, and S. ten Brink, "Extrinsic information transfer functions: model and erasure channel properties," IEEE Trans. Inf. Theory, vol. 50, pp. 2657-2673, Nov. 2004.

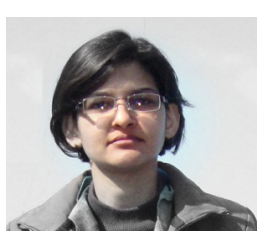

Zunaira Babar received her B.Eng. degree in electrical engineering from the National University of Science \& Technology (NUST), Islamabad, Pakistan, in 2008 and M.Sc. degree (Distinction) in wireless communications from the University of Southampton, UK, in 2011. She is currently pursuing her PhD degree in the Communications, Signal Processing and Control Group, School of Electronics and Computer Science, University of Southampton, UK. Her research interests include quantum error correction codes, channel coding, coded modulation,

iterative detection and cooperative communications.

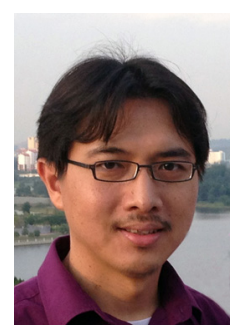

Dr. Soon Xin Ng (S'99-M'03-SM'08) received the B.Eng. degree (First class) in electronics engineering and the Ph.D. degree in wireless communications from the University of Southampton, Southampton, U.K., in 1999 and 2002, respectively. From 2003 to 2006, he was a postdoctoral research fellow working on collaborative European research projects known as SCOUT, NEWCOM and PHOENIX. Since August 2006, he has been a member of academic staff in the School of Electronics and Computer Science, University of Southampton. He is involved in the OPTIMIX and CONCERTO European projects as well as the IU-ATC and UC4G projects. He is currently a senior lecturer at the University of Southampton.

His research interests include adaptive coded modulation, coded modulation, channel coding, space-time coding, joint source and channel coding, iterative detection, OFDM, MIMO, cooperative communications, distributed coding, quantum error correction codes and joint wireless-and-optical-fiber communications. He has published over 160 papers and co-authored two John Wiley/IEEE Press books in this field. He is a Senior Member of the IEEE, a Chartered Engineer and a Fellow of the Higher Education Academy in the UK.

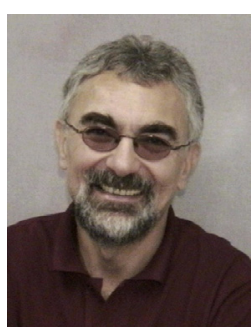

Lajos Hanzo (http://www-mobile.ecs.soton.ac.uk) FREng, FIEEE, FIET, Fellow of EURASIP, DSc received his degree in electronics in 1976 and his doctorate in 1983. In 2009 he was awarded the honorary doctorate "Doctor Honoris Causa" by the Technical University of Budapest. During his 37-year career in telecommunications he has held various research and academic posts in Hungary, Germany and the UK. Since 1986 he has been with the School of Electronics and Computer Science, University of Southampton, UK, where he holds the chair in telecommunications. He has successfully supervised $80+\mathrm{PhD}$ students, co-authored 20 John Wiley/IEEE Press books on mobile radio communications totalling in excess of 10000 pages, published 1300+ research entries at IEEE Xplore, acted both as TPC and General Chair of IEEE conferences, presented keynote lectures and has been awarded a number of distinctions. Currently he is directing a 100-strong academic research team, working on a range of research projects in the field of wireless multimedia communications sponsored by industry, the Engineering and Physical Sciences Research Council (EPSRC) UK, the European Research Council's Advanced Fellow Grant and the Royal Society's Wolfson Research Merit Award. He is an enthusiastic supporter of industrial and academic liaison and he offers a range of industrial courses. He is also a Governor of the IEEE VTS. During 2008 - 2012 he was the Editor-in-Chief of the IEEE Press and a Chaired Professor also at Tsinghua University, Beijing. His research is funded by the European Research Council's Senior Research Fellow Grant. For further information on research in progress and associated publications please refer to http://www-mobile.ecs.soton.ac.uk Lajos has 18 000+ citations. 